\title{
Gastrointestinal Complaints During Exercise: Prevalence, Etiology, and Nutritional Recommendations
}

\author{
Erick Prado de Oliveira • Roberto Carlos Burini • \\ Asker Jeukendrup
}

(C) The Author(s) 2014. This article is published with open access at Springerlink.com

\begin{abstract}
Gastrointestinal problems are common, especially in endurance athletes, and often impair performance or subsequent recovery. Generally, studies suggest that $30-50 \%$ of athletes experience such complaints. Most gastrointestinal symptoms during exercise are mild and of no risk to health, but hemorrhagic gastritis, hematochezia, and ischemic bowel can present serious medical challenges. Three main causes of gastrointestinal symptoms have been identified, and these are either physiological, mechanical, or nutritional in nature. During intense exercise, and especially when hypohydrated, mesenteric blood flow is reduced; this is believed to be one of the main contributors to the development of gastrointestinal symptoms. Reduced splanchnic perfusion could result in compromised gut permeability in athletes. However, although evidence exists that this might occur, this has not yet been definitively linked to the prevalence of gastrointestinal symptoms. Nutritional training and appropriate nutrition choices can reduce the risk of gastrointestinal discomfort
\end{abstract}

E. P. de Oliveira $(\bowtie)$

School of Medicine, Federal University of Uberlândia, Av. Pará, no 1720 Bloco 2U, Campus Umuarama, Uberlândia,

Minas Gerais 38400-902, Brazil

e-mail: erick_po@yahoo.com.br

R. C. Burini

Centre for Physical Exercise and Nutrition Metabolism, UNESP

School of Medicine, Public Health Department, Botucatu,

São Paulo, Brazil

A. Jeukendrup

Gatorade Sports Science Institute, Barrington, IL, USA

A. Jeukendrup

School of Sport, Exercise and Health Sciences, Loughborough

University, Loughborough, UK during exercise by ensuring rapid gastric emptying and the absorption of water and nutrients, and by maintaining adequate perfusion of the splanchnic vasculature. A number of nutritional manipulations have been proposed to minimize gastrointestinal symptoms, including the use of multiple transportable carbohydrates, and potentially the use of nutrients that stimulate the production of nitric oxide in the intestine and thereby improve splanchnic perfusion. However, at this stage, evidence for beneficial effects of such interventions is lacking, and more research needs to be conducted to obtain a better understanding of the etiology of the problems and to improve the recommendations to athletes.

\section{Introduction}

Gastrointestinal complaints are very common among endurance athletes. Anecdotally, gastrointestinal problems are perhaps the most common cause of underperformance in endurance events. Depending on the methodology used and the events studied, an estimated 30-90\% of distance runners experience intestinal problems related to exercise $[1,2]$. These complaints may be of varying severity, but symptoms may include nausea, vomiting, abdominal angina, and bloody diarrhea. In many cases, these problems have not only negative effects on performance but also an impact on subsequent recovery. The presence and nature of abdominal symptoms experienced by athletes vary from mild, exercise-related discomfort to severe ischemic colitis and diarrhea [3]. Bill Rodgers, marathon legend, with four victories in both the Boston marathon and the New York City marathon in the late 1970s said, "More marathons are won or lost in the porta-toilets than at the dinner table." This illustrates the magnitude of the problem for endurance 
athletes, particularly long-distance runners. This review discusses the prevalence of gastrointestinal complaints in athletes and the etiology of the problems, and begins to develop guidelines to prevent the issues.

\section{Methodological Considerations}

Clearly, there is large variation in the reported prevalence in the literature and this seems to be attributable to, among other factors, the method of investigation (and the way in which gastrointestinal symptoms are defined and recorded). However, in addition, the reported prevalence of these symptoms varies in different studies depending on the study population, sex, age, and training status of the athletes, as well as the mode and intensity of the exercise studied, and the environmental conditions. There is also no consistent definition of what constitutes a severe or nonsevere symptom. It has been suggested that a severe symptom is any symptom that impacts performance and/or health. So, for example, very mild nausea may not affect performance, but a higher degree of nausea is likely to have negative consequences. Flatulence is unlikely to have effects on performance, but vomiting, independent of the severity score provided by the athlete, is likely to have a negative impact on performance. This is an approach discussed by the authors in a paper in 2000 [2] and it is suggested that future studies in this area use a similar categorization of symptoms and scoring approach.

When analyzing the reported symptoms, it becomes immediately obvious that they are highly individual and there are no clear patterns with regard to the type of activity and the types of symptoms observed. There is a fairly large, yet well defined number of different gastrointestinal symptoms that can occur during exercise. Generally, the symptoms can be classified as either upper or lower gastrointestinal tract. Typically, lower gastrointestinal tract problems are more severe in nature, but all symptoms have the potential to impair performance. Symptoms are often mild and may not affect performance, but they can also be very serious and can not only affect performance, but also be health threatening.

\section{Prevalence of Gastrointestinal Symptoms in Athletes}

Gastrointestinal symptoms are common in many sports but particularly in endurance events. One early review stated that in exhausting endurance events, 30-50\% of participants may experience one or more gastrointestinal symptoms [1]. A study in long-distance triathletes who competed in extreme conditions demonstrated a prevalence of up to $93 \%$ for any gastrointestinal symptom [2]. Among elite endurance athletes, the prevalence of exercise-induced gastrointestinal symptoms was reported to be $70 \%$ [4], and in an internet-based observational study of 1,281 athletes, $45 \%$ reported at least one gastrointestinal symptom [5]. Gastrointestinal distress is a pervasive problem, especially in ultra-endurance events [6-9]. Nausea, vomiting, abdominal cramping, and diarrhea have been reported in $37-89 \%$ of runners participating in races $67-161 \mathrm{~km}$ long [6-9], and fecal blood loss indicating gastrointestinal hemorrhage was reported in $85 \%$ of participants in a $161 \mathrm{~km}$ ultra-marathon [6]. A recent study investigated gastrointestinal problems in a group of ultra-marathon runners, and observed that 9 of 15 runners experienced gastrointestinal distress, including nausea ( $89 \%$ ), abdominal cramps (44\%), diarrhea (44\%), and vomiting (22\%) [9].

The prevalence of symptoms varies considerably depending on the event, the environmental conditions, and the level of the athlete. Pfeiffer et al. [10] reported severe gastrointestinal distress ranging from $4 \%$ in marathon running and cycling up to $32 \%$ in Ironman races. Large differences were observed not only between the different events, but also between individuals within the same event. It was demonstrated that there was a strong correlation between gastrointestinal complaints and having a history of gastrointestinal symptoms, indicating that some people are more prone to developing gastrointestinal symptoms [10, $11]$, and suggesting that there is a large genetic component to these problems.

Gastrointestinal symptoms are not only inconvenient, they can also affect performance and, in extreme cases, have longer-term health implications. In one study, $43 \%$ of triathletes reported serious gastrointestinal problems, and $7 \%$ abandoned the race because of gastrointestinal problems [2]. In two 161-km ultra-marathons, nausea and/ or vomiting were the main reasons for dropping out among non-finishers and were the second most common problem impacting race performance among finishers [7]. Clearly, gastrointestinal problems can have major effects on performance.

\section{Gastrointestinal Symptoms and Pathophysiology}

Among the reported deleterious manifestations of strenuous exercise are mucosal erosions and ischemic colitis, both observed after long-distance running [12-14]. For example, marathon runners and long-distance triathletes occasionally have blood loss in the feces in the hours following a marathon. Schaub et al. [15] observed epithelial surface changes known to occur during ischemia on colonoscopic inspection of one such triathlete following a marathon, and suggested that ischemia of the lower 
gastrointestinal tract induced the problems. Blood loss as a result of ischemic colitis is not uncommon in athletes, and can be profound in extreme cases. Proximal, distal, or pancolitis, and even small bowel, infarctions have been reported in athletes and in some cases have required surgery $[14,16]$. Despite the high prevalence of mild or severe symptoms, the etiology of these gastrointestinal complaints in endurance athletes is still incompletely understood.

\section{Causes of Gastrointestinal Problems}

While it is recognized that the etiology of exercise-induced gastrointestinal distress is multifactorial, gastrointestinal ischemia is often acknowledged as the main pathophysiological mechanism for the emergence of the symptoms [5, $17,18]$. The other factors are mechanical and nutritional in nature.

\subsection{Effects of Exercise on Gut Function}

The effects of exercise on gastrointestinal function have been studied in different ways. Traditionally, the research focused on gut perfusion, but more recently the effects of exercise on motility, gut barrier function, and absorption have been studied. Having an understanding of the physiology of the gut during exercise is essential to elucidating the factors that may contribute to the development of gastrointestinal symptoms.

\subsubsection{Splanchnic Hypoperfusion}

Large heterogeneity exists in the response of the gastrointestinal system to exercise. Splanchnic hypoperfusion during exercise ranges from mild circulatory changes to profound gastrointestinal ischemia [19]. In line with this, the consequences of hypoperfusion within the gastrointestinal tract (i.e., epithelial injury and changes in gastrointestinal permeability and epithelial barrier function) differ greatly between individuals. During strenuous physical activity or exercise, norepinephrine is released from nerve endings and on binding to $\alpha$-adrenoreceptors of the sympathetic nervous system, induces splanchnic vasoconstriction. This will result in an increase in the total splanchnic vascular resistance [20, 21], while, at the same time, vascular resistance with increased activity during exercise in other tissues, such as the heart, lungs, active muscle, and skin, is decreased [22, 23]. During maximal exercise, splanchnic blood flow may be reduced by up to $80 \%$ to provide sufficient blood flow to working muscle and skin. As blood is shunted from viscera to the active tissues [23], gut mucosal ischemia may result, as well as increases in mucosal permeability [19, 24]. This in turn may be linked to nausea, vomiting, abdominal pain, and diarrhea [17, 25], although convincing evidence for this is lacking [26].

\subsubsection{Changes in Motility}

Changes in motility might be observed at different levels of the intestinal tract: the esophagus, the stomach, and the intestine. Decreases in esophageal peristaltic activity, a decrease in lower esophageal sphincter tone, and increased transient lower sphincter relaxation have been observed and could be linked to gastro-esophageal reflux during exercise [27]. The effects on gastric emptying are less clear, but several studies, including a very early study [28], reported no effect of moderate exercise on gastric emptying. However, during exercise at very high intensity or during intermittent activity, gastric emptying may be affected [29].

When gastric emptying was studied using the Loughborough intermittent shuttle test, gastric emptying of fluids was reduced approximately by half. Exercising in the heat per se did not appear to affect gastric emptying very much, except at extreme temperatures $\left(49^{\circ} \mathrm{C}\right)$ [30]. However, exercising in a hypohydrated state does seem to significantly affect gastric emptying [30, 31].

Studies performed so far suggest that the effects of exercise on the small bowel as well as on the colon are limited. In one study, motility was investigated in symptomatic and asymptomatic runners using a telemetric $\mathrm{pH}$ sensor [32]. It was observed that small bowel and colonic transit times were similar in the two groups at rest. Interestingly, although diarrhea was reported in that study, there was no link with colonic transit time and therefore there must be another cause for the observed gastrointestinal symptoms.

Overall, it seems that moderate exercise has little effect on gastrointestinal tract motility, but when exercise becomes more severe, there may be some inhibiting effects, especially at the level of gastric emptying.

\subsubsection{Absorption and Gut Permeability}

Studies also suggest that exercise has little effect on intestinal absorption of both water and carbohydrate [33, 34]. However, it must be noted that most studies used exercise intensities that were moderate and durations of exercise that were no longer than $2 \mathrm{~h}$. It is feasible that, during higher intensities of exercise when intestinal blood flow is more compromised, and also after more prolonged exercise, that absorption could be reduced. It has also been reported that with fluid restriction intestinal permeability may be increased [35], possibly because dehydration ultimately influences gut perfusion. 
Oktedalen et al. [36] reported increased intestinal permeability after a marathon, indicating damage to the gut and impaired gut function. There are numerous techniques available to study gut permeability but to date there is limited information. The information that is available suggests that gut permeability can be compromised in athletes [24]. Although this has not been conclusively linked to gastrointestinal symptoms, one study showed that gut permeability in symptomatic runners was greater than in asymptomatic runners [37]. On the other hand, in one long-distance triathlon in extreme conditions in which gastrointestinal symptoms were highly prevalent, no compromised gut barrier function was observed as measured by bacterial translocation (lipopolysaccharide stimulation). This technique is a marker of mucosal damage and the invasion of Gram-negative intestinal bacteria and/or their toxic constituents (endotoxins) into the blood circulation [2]. More research needs to be conducted before there is a clear understanding of the causes of gastrointestinal distress.

\subsubsection{Summary of the Effects of Exercise on Gut Function}

In summary, exercise results in numerous changes in the intestinal tract, and most of these effects are intensity dependent. Many of the functions are not affected at lowintensity exercise but become progressively affected at higher intensities. The changes include a reduction in mesenteric blood flow, decreases in esophageal peristaltic activity, decreases in lower esophageal sphincter tone, and increased transient lower sphincter time and a reduction of gastric emptying. As a result of reduced gastrointestinal tract perfusion, absorption might be affected and gut barrier function might be compromised.

\subsection{Mechanical Causes}

The mechanical causes of gastrointestinal problems are related to either impact or posture. For example, symptoms are more common in runners than in cyclists [27]. This is thought to be a result of the repetitive high-impact mechanics of running and subsequent damage to the intestinal lining [38]. This repetitive gastric jostling is also thought to contribute to lower gastrointestinal symptoms such as flatulence, diarrhea, and urgency. The mechanical trauma suffered by the gut from the repetitive impact of running in combination with gut ischemia is probably the cause of the bleeding [3, 25].

Posture can also have an effect on gastrointestinal symptoms. For example, on a bicycle, upper gastrointestinal symptoms are more prevalent possibly due to increased pressure on the abdomen as a result of the cycling position, particularly when in the 'aero' position.
'Swallowing' air as a result of increased respiration and drinking from water bottles can result in mild to moderate stomach distress. In general, the only way to reduce the effects of these mechanical causes is by training [3, 39].

\subsection{Nutritional Causes}

It is known that nutrition can have a strong influence on gastrointestinal distress, although many of the problems can occur in the absence of any food intake before or during exercise. Fiber, fat, protein, and fructose have all been associated with a greater risk of developing gastrointestinal symptoms. Dehydration, possibly as a result of inadequate fluid intake, may also exacerbate the symptoms. A study by Rehrer et al. [40] demonstrated a link between nutritional practices and gastrointestinal complaints during a half-Ironman triathlon. Gastrointestinal problems were more likely to occur with the ingestion of fiber, fat, protein, and concentrated carbohydrate solutions during the triathlon. Beverages with high osmolalities (>500 mOsm/L) seemed to be associated with an increased incidence of symptoms.

It appears that foods that delay gastric emptying and might cause a shift of fluids into the intestinal lumen are more likely to cause gastrointestinal symptoms. Highly concentrated carbohydrate solutions with high osmolalities could have this effect. Although subject numbers were too small to perform meaningful statistical analysis, a study by Wallis et al. [41] reported more severe gastrointestinal symptoms in women with a high carbohydrate intake $(1.0$ or $1.5 \mathrm{~g} / \mathrm{min}$ ) than in those with a low intake $(0$ or $0.5 \mathrm{~g} /$ $\min )$. However, the data are equivocal. For example, in a field study in which runners ran two 16-mile races while consuming carbohydrate at a high rate $(1.4 \mathrm{~g} / \mathrm{min})$ in the form of gels, very few gastrointestinal symptoms were observed ( $\sim 10 \%$ of runners reported symptoms) [11]. Perhaps the duration of the exercise was not long enough to cause significant gastrointestinal distress, or, alternatively, carbohydrate content per se is not as important a factor as has often been assumed. In a larger study, with 221 endurance athletes competing in various events including marathons and Ironman races, carbohydrate intake was positively correlated to nausea and flatulence [10]. However, nausea was relatively mild and a higher carbohydrate intake was also correlated with faster finish times, suggesting that nausea did not have any negative effects on performance. Of course, no causal relationships can be obtained from these correlations so the data must be interpreted with caution.

It may be speculated that it is not simply carbohydrate intake that causes gastrointestinal symptoms, but it may be a complex interplay of a number of factors such as carbohydrate concentration, the type of carbohydrate, 
osmolality and acidity of a beverage that might be linked with gastrointestinal problems. More research is definitely needed to find carbohydrate solutions that reduce the risk of developing gastrointestinal symptoms.

\section{Nutritional Solutions to Gastrointestinal Problems}

Although much is still unknown about the etiology of gastrointestinal symptoms, a number of nutritional manipulations have been suggested to reduce the number or the severity of symptoms. For athletes who compete in endurance events, an interesting observation is that when a beverage is consumed that contains multiple transportable carbohydrates such as glucose and fructose, gastrointestinal symptoms seem to be reduced compared with the consumption of the same (large) amount of a single carbohydrate (glucose). This has been a consistent finding in a number of studies from the authors' laboratory [42-45]. Rowlands et al. [46] recently reported fewer gastrointestinal problems with multiple transportable carbohydrates (maltodextrin : fructose) in mountain bikers, and concluded that the ingestion of multiple transportable compares with a single carbohydrate-enhanced mountain bike race and high-intensity laboratory cycling performance. These results were found with inconsistent, but not irreconcilable, effects of gut discomfort as a possible mediating mechanism.

More recently, van Wijck et al. [19] argued that, if perfusion of the gut is one of the main causes of the problems, then manipulation of blood flow to the gut by upregulating nitric oxide production could be a way to reduce symptoms. There are a number of options to upregulate intestinal nitric oxide availability that may be of value for athletes experiencing abdominal distress associated with splanchnic hypoperfusion, including nitric oxide synthase-dependent (glutamine-arginine-citrulline) and nitric oxide synthase-independent (nitrate-nitrite) supplementation. Research is in its infancy, but of the ingredients that have been studied, dietary nitrate probably has the most potential to influence splanchnic perfusion [19]. However, current recognized vegetable sources of dietary nitrate are also sometimes associated with gastrointestinal symptoms.

\section{7 'Training the Gut'}

For many years, it has been suggested that the gut can be 'conditioned' or 'trained' in order to reduce gastrointestinal distress, yet research in this area is still in its infancy [47, 48]. It has been shown that athletes who are not accustomed to fluid and food ingestion during exercise had a twofold risk of developing gastrointestinal symptoms than athletes who were accustomed to taking fluid and food during exercise [5]. In a study by Cox et al. [49], the adaptability of the gut was nicely demonstrated. In that study, 16 endurance-trained cyclists or triathletes were pair matched and randomly allocated to either a high carbohydrate group (high group; $n=8$ ) or an energy-matched low carbohydrate group (low group; $n=8$ ) for 28 days. It became apparent after 28 days that the high carbohydrate group had higher exogenous carbohydrate oxidation rates during exercise [49]. The higher rates were attributed to improved absorption, which is generally associated with improved tolerance of fluids and foods during exercise, and thus the training would have reduced the chances of gastrointestinal distress [48].

However, it also appears that training with a nutrition strategy can improve tolerance and stomach comfort, independent of changes in adaptations in gut function. Lambert et al. [50] assessed tolerance to fluid ingestion with repeated sessions of drinking while running. During five runs, subjects drank a volume of the solution every $10 \mathrm{~min}$ equal to their sweat production over $10 \mathrm{~min}$. Although repeated sessions of drinking at a rate matching sweat rate improved stomach comfort, the gastric emptying rate did not change under such conditions.

More research is needed to understand the adaptations that occur in the intestinal tract, as well as the time course of such changes. This is essential to develop guidance for athletes on how often certain nutritional strategies need to be practised and the best way to conduct such training sessions.

\section{Non-Steroidal Anti-Inflammatory Drugs}

Large numbers of athletes have reported using analgesics to relieve existing or anticipated pain [51]. The use of non-selective non-steroidal anti-inflammatory drugs (NSAIDs) has been associated with a three- to fivefold increased risk of upper gastrointestinal complications, mucosal bleeding, or perforation compared with no medication [52]. In a study at the Chicago marathon [53], it was found that ibuprofen (but not aspirin) ingestion during prolonged exercise may have increased gastrointestinal permeability and led to gastrointestinal symptoms. van Wijck et al. [54] recently demonstrated that ibuprofen aggravates exercise-induced small intestinal injury and induces gut barrier dysfunction, and concluded that the consumption of NSAIDs by athletes is not harmless and should be discouraged in those who experience persistent or recurring gastrointestinal symptoms. 


\section{Practical Implications and Advice to Athletes}

In order to prevent gastrointestinal distress, a few guidelines can be provided. However, it must be noted that these are based on limited research. Nevertheless, anecdotally, these guidelines seem to be effective:

- Avoid high-fiber foods in the day or even days before competition. For the athlete in training, a diet with adequate fiber will help keep the bowel regular.

- Avoid aspirin and NSAIDs such as ibuprofen. Both aspirin and NSAIDs have commonly been shown to increase intestinal permeability and may increase the incidence of gastrointestinal complaints. The use of NSAIDs in the pre-race period should be discouraged, mainly for athletes with a history of gastrointestinal problems.

- Avoid high-fructose foods (in particular drinks that are exclusively fructose). However, interestingly, a fructose and glucose combination may not cause problems and may be better tolerated.

- Avoid dehydration. As dehydration can exacerbate gastrointestinal symptoms, it is important to prevent dehydration. Start the race (or training) well hydrated.

- Ingest carbohydrates with sufficient water or choose drinks with lower carbohydrate concentrations to prevent very high concentrations and osmolalities in the stomach.

- Practise new nutrition strategies. Make sure to experiment with the pre-race and race-day nutrition plan many times before the race day. This will allow the athlete to work out what does and does not work, and reduces the chances of getting gastrointestinal symptoms.

\section{Conclusions}

The gut is an important athletic organ because it is responsible for the delivery of water and nutrients during exercise. Both upper and lower gastrointestinal complaints are highly prevalent among athletes during exercise (especially endurance athletes), and can negatively impact performance. In severe cases, health risks can also be present. Most gastrointestinal complaints during exercise are mild and of no risk to health, but hemorrhagic gastritis, hematochezia, and ischemic bowel can present serious medical challenges. Nutritional training and appropriate nutritional choices (avoiding protein, fat, fiber, and milk products) can reduce the risk of gastrointestinal discomfort during exercise by ensuring rapid gastric emptying and the absorption of water and nutrients and by maintaining adequate perfusion of the splanchnic vasculature.
Acknowledgments This article was published in a supplement supported by Gatorade Sports Science Institute. The supplement was guest edited by Lawrence L. Spriet who attended a meeting of the Gatorade Sports Science Institute (GSSI) expert panel in April 2012 and received honoraria from the GSSI, a division of PepsiCo, Inc., for his meeting participation and the writing of his manuscript. He has not received any honoraria for guest editing the supplement. L.L.S. selected peer reviewers for each paper and managed the process. Erick de Oliveira $\mathrm{PhD}$ attended a meeting of the Gatorade Sports Science Institute (GSSI) Expert Panel in April 2012 and received honoraria from the GSSI, a division of PepsiCo, Inc., for his meeting participation and the writing of this manuscript. Asker Jeukendrup is an employee of the GSSI. The views expressed in this manuscript are those of the authors and do not necessarily reflect the position or policy of PepsiCo, Inc. Roberto Burini has no conflict of interest.

Open Access This article is distributed under the terms of the Creative Commons Attribution License which permits any use, distribution, and reproduction in any medium, provided the original author(s) and the source are credited.

\section{References}

1. Brouns F, Beckers E. Is the gut an athletic organ? Digestion, absorption and exercise. Sports Med. 1993;15:242-57.

2. Jeukendrup AE, Vet-Joop K, Sturk A, et al. Relationship between gastro-intestinal complaints and endotoxaemia, cytokine release and the acute-phase reaction during and after a long-distance triathlon in highly trained men. Clin Sci (Lond). 2000;98:47-55.

3. Moses FM. The effect of exercise on the gastrointestinal tract. Sports Med. 1990;9:159-72.

4. Peters HP, Bos M, Seebregts L, et al. Gastrointestinal symptoms in long-distance runners, cyclists, and triathletes: prevalence, medication, and etiology. Am J Gastroenterol. 1999;94:1570-81.

5. ter Steege RW, Van der Palen J, Kolkman JJ. Prevalence of gastrointestinal complaints in runners competing in a long-distance run: an internet-based observational study in 1281 subjects. Scand J Gastroenterol. 2008;43:1477-82.

6. Baska RS, Moses FM, Graeber G, et al. Gastrointestinal bleeding during an ultramarathon. Dig Dis Sci. 1990;35:276-9.

7. Hoffman MD, Fogard K. Factors related to successful completion of a 161-km ultramarathon. Int J Sports Physiol Perform. 2011;6:25-37.

8. Rehrer NJ, Brouns F, Beckers EJ, et al. Physiological changes and gastro-intestinal symptoms as a result of ultra-endurance running. Eur J Appl Physiol. 1992;64:1-8.

9. Stuempfle KJ, Hoffman MD, Hew-Butler T. Gastrointestinal distress in ultramarathoners is associated with race diet. Int $\mathbf{J}$ Sport Nutr Exerc Metab. 2013;23:103-9.

10. Pfeiffer B, Stellingwerff T, Hodgson AB, et al. Nutritional intake and gastrointestinal problems during competitive endurance events. Med Sci Sports Exerc. 2012;44:344-51.

11. Pfeiffer B, Cotterill A, Grathwohl D, et al. The effect of carbohydrate gels on gastrointestinal tolerance during a $16-\mathrm{km}$ run. Int J Sport Nutr Exerc Metab. 2009;19:485-503.

12. Choi SC, Choi SJ, Kim JA, et al. The role of gastrointestinal endoscopy in long-distance runners with gastrointestinal symptoms. Eur J Gastroenterol Hepatol. 2001;13:1089-94.

13. Moses FM. Exercise-associated intestinal ischemia. Curr Sports Med Rep. 2005;4:91-5.

14. Heer M, Repond F, Hany A, et al. Acute ischaemic colitis in a female long distance runner. Gut. 1987;28:896-9.

15. Schaub N, Spichtin HP, Stalder GA. Ischemic colitis as a cause of intestinal bleeding after marathon running. Schweiz Med Wochenschr. 1985;115:454-7. 
16. Lucas W, Schroy PC III. Reversible ischemic colitis in a high endurance athlete. Am J Gastroenterol. 1998;93:2231-4.

17. de Oliveira EP, Burini RC. Food-dependent, exercise-induced gastrointestinal distress. J Int Soc Sports Nutr. 2011;8:12.

18. ter Steege RW, Geelkerken RH, Huisman AB, et al. Abdominal symptoms during physical exercise and the role of gastrointestinal ischaemia: a study in 12 symptomatic athletes. Br J Sports Med. 2012;46:931-5.

19. van Wijck K, Lenaerts K, Grootjans J, et al. Physiology and pathophysiology of splanchnic hypoperfusion and intestinal injury during exercise: strategies for evaluation and prevention. Am J Physiol. 2012;303:G155-68.

20. Wright H, Collins M, Villiers RD, et al. Are splanchnic hemodynamics related to the development of gastrointestinal symptoms in ironman triathletes? A prospective cohort study. Clin J Sport Med. 2011;21:337-43.

21. Otte JA, Geelkerken RH, Oostveen E, et al. Clinical impact of gastric exercise tonometry on diagnosis and management of chronic gastrointestinal ischemia. Clin Gastroenterol Hepatol. 2005;3:660-6.

22. Otte JA, Oostveen E, Geelkerken RH, et al. Exercise induces gastric ischemia in healthy volunteers: a tonometry study. J Appl Physiol. 2001;91:866-71.

23. Qamar MI, Read AE. Effects of exercise on mesenteric blood flow in man. Gut. 1987;28:583-7.

24. Pals KL, Chang RT, Ryan AJ, et al. Effect of running intensity on intestinal permeability. J Appl Physiol. 1997;82:571-6.

25. de Oliveira EP, Burini RC. The impact of physical exercise on the gastrointestinal tract. Curr Opin Clin Nutr Metab Care. 2009;12:533-8.

26. ter Steege RW, Kolkman JJ. Review article: the pathophysiology and management of gastrointestinal symptoms during physical exercise, and the role of splanchnic blood flow. Aliment Pharmacol Ther. 2012;35:516-28.

27. Peters HP, Wiersma JW, Koerselman J, et al. The effect of a sports drink on gastroesophageal reflux during a run-bike-run test. Int J Sports Med. 2000;21:65-70.

28. Fordtran JS, Saltin B. Gastric emptying and intestinal absorption during prolonged severe exercise. J Appl Physiol. 1967;23:331-5.

29. Leiper JB, Prentice AS, Wrightson C, et al. Gastric emptying of a carbohydrate-electrolyte drink during a soccer match. Med Sci Sports Exerc. 2001;33:1932-8.

30. Neufer PD, Young AJ, Sawka MN. Gastric emptying during exercise: effects of heat stress and hypohydration. Eur J Appl Physiol Occup Physiol. 1989;58:433-9.

31. Rehrer NJ, Beckers EJ, Brouns F, et al. Effects of dehydration on gastric emptying and gastrointestinal distress while running. Med Sci Sports Exerc. 1990;22:790-5.

32. Rao KA, Yazaki E, Evans DF, et al. Objective evaluation of small bowel and colonic transit time using $\mathrm{pH}$ telemetry in athletes with gastrointestinal symptoms. Br J Sports Med. 2004;38:482-7.

33. Ryan AJ, Lambert GP, Shi X, et al. Effect of hypohydration on gastric emptying and intestinal absorption during exercise. J Appl Physiol. 1998;84:1581-8.

34. Lambert GP, Chang RT, Xia T, et al. Absorption from different intestinal segments during exercise. J Appl Physiol. 1997;83:204-12.

35. Lambert GP, Lang J, Bull A, et al. Fluid restriction during running increases GI permeability. Int J Sports Med. 2008;29:194-8.
36. Oktedalen O, Lunde OC, Opstad PK, et al. Changes in the gastrointestinal mucosa after long-distance running. Scand J Gastroenterol. 1992;27:270-4.

37. van Nieuwenhoven MA, Brouns F, Brummer RJ. Gastrointestinal profile of symptomatic athletes at rest and during physical exercise. Eur J Appl Physiol. 2004;91:429-34.

38. Rudzki SJ, Hazard H, Collinson D. Gastrointestinal blood loss in triathletes: its etiology and relationship to sports anaemia. Aust J Sci Med Sport. 1995;27:3-8.

39. Waterman JJ, Kapur R. Upper gastrointestinal issues in athletes. Curr Sports Med Rep. 2012;11:99-104.

40. Rehrer NJ, van Kemenade M, Meester W, et al. Gastrointestinal complaints in relation to dietary intake in triathletes. Int J Sport Nutr. 1992;2:48-59.

41. Wallis GA, Yeo SE, Blannin AK, et al. Dose-response effects of ingested carbohydrate on exercise metabolism in women. Med Sci Sports Exerc. 2007;39:131-8.

42. Jentjens RL, Underwood K, Achten J, et al. Exogenous carbohydrate oxidation rates are elevated after combined ingestion of glucose and fructose during exercise in the heat. J Appl Physiol. 2006;100:807-16.

43. Jeukendrup AE, Moseley L, Mainwaring GI, et al. Exogenous carbohydrate oxidation during ultraendurance exercise. J Appl Physiol. 2006;100:1134-41.

44. Jentjens RL, Achten J, Jeukendrup AE. High oxidation rates from combined carbohydrates ingested during exercise. Med Sci Sports Exerc. 2004;36:1551-8.

45. Jentjens RL, Venables MC, Jeukendrup AE. Oxidation of exogenous glucose, sucrose, and maltose during prolonged cycling exercise. J Appl Physiol. 2004;96:1285-91.

46. Rowlands DS, Swift M, Ros M, et al. Composite versus single transportable carbohydrate solution enhances race and laboratory cycling performance. Appl Physiol Nutr Metab. 2012;37:425-36.

47. Murray R. Training the gut for competition. Curr Sports Med Rep. 2006;5:161-4.

48. Jeukendrup AE, McLaughlin J. Carbohydrate ingestion during exercise: effects on performance, training adaptations and trainability of the gut. Nestle Nutr Inst Workshop Ser. 2011;69:1-12 (discussion 13-7).

49. Cox GR, Clark SA, Cox AJ, et al. Daily training with high carbohydrate availability increases exogenous carbohydrate oxidation during endurance cycling. J Appl Physiol. 2010;109:126-34.

50. Lambert GP, Lang J, Bull A, et al. Fluid tolerance while running: effect of repeated trials. Int J Sports Med. 2008;29:878-82.

51. Gorski T, Cadore EL, Pinto SS, et al. Use of NSAIDs in triathletes: prevalence, level of awareness and reasons for use. $\mathrm{Br} \mathrm{J}$ Sports Med. 2011;45:85-90.

52. Gabriel SE, Jaakkimainen L, Bombardier C. Risk for serious gastrointestinal complications related to use of nonsteroidal antiinflammatory drugs. A meta-analysis. Ann Intern Med. 1991;115:787-96.

53. Smetanka RD, Lambert GP, Murray R, et al. Intestinal permeability in runners in the 1996 Chicago marathon. Int J Sport Nutr. 1999;9:426-33.

54. van Wijck K, Lenaerts K, van Bijnen AA, et al. Aggravation of exercise-induced intestinal injury by Ibuprofen in athletes. Med Sci Sports Exerc. 2012;44:2257-62. 